\title{
CITED BRAZILIAN PAPERS IN GENERAL SURGERY BETWEEN 1970 AND 2009
}

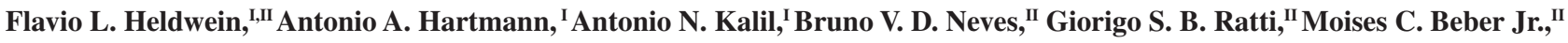 \\ Rafael M. Souza, ${ }^{\text {II }}$ Armando J. d'Acampora ${ }^{\text {II }}$
}

doi: $10.1590 / \mathbf{S 1 8 0 7 - 5 9 3 2 2 0 1 0 0 0 0 5 0 0 0 1 0}$

\author{
Heldwein FL, Hartmann AA, Kalil AN, Neves BVD, Ratti GSB, Beber-Junior MC, Souza RM, d’Acampora AJ. Cited Brazilian \\ papers in general surgery between 1970 and 2009. Clinics. 2010;65(5):521-9.
}

OBJECTIVES: To identify the most cited articles in general surgery published by Brazilian authors.

INTRODUCTION: There are several ways for the international community to recognize the quality of a scientific article. Although controversial, the most widely used and reliable methodology to identify the importance of an article is citation analysis.

METHODS: A search using the Institute for Scientific Information citation database (Science Citation Index Expanded) was performed to identify highly cited Brazilian papers published in twenty-six highly cited general surgery journals, selected based on their elevated impact factors, from 1970 to 2009. Further analysis was done on the 65 most-cited papers.

RESULTS: We identified 1,713 Brazilian articles, from which nine papers emerged as classics (more than 100 citations received). For the Brazilian contributions, a total increase of about 21-fold was evident between 1970 and 2009. Although several topics were covered, articles covering trauma, oncology and organ transplantation were the most cited. The majority of classic studies were done with international cooperation.

CONCLUSIONS: This study identified the most influential Brazilian articles published in internationally renowned general surgery journals.

KEYWORDS: Peer review; Surgery/history; Classical article; Surgery/statistics and numerical data

\section{INTRODUCTION}

A central aspect of general surgery is the diversity of topics and domains. General surgery has become one of the most well-known medical specialties. At present, general surgery boasts remarkable developments and refinements in basic science, diagnosis and treatment that have transcended abdominal organs, touching neighboring and more distal branches of medicine. Advances in scientific surgical research have benefited the care of men and women of all ages.

\footnotetext{
${ }^{\text {I }}$ Department of Pathology, Universidade Federal de Ciências da Saúde (UFCSPA) - Porto Alegre/RS, Brazil.

II Department of Surgery, Universidade do Sul de Santa Catarina (UNISUL)

- Florianópolis/SC, Brazil.

Email: flavio.lobo@gmail.com

Tel.: 5548 3223-0816

Received for publication on November 28, 2009

First review completed December 28, 2009

Accepted for publication on February 09, 2010
}

A scientific article's impact is established over time. Because publications inspire future articles, a paper's scientific impact can be measured in terms of the number of citations received. ${ }^{1}$ Increasing numbers of scientific papers in the Internet era have provided diverse information. The frequency of citations serves as a rough guide to the importance of an article. The impact factor (IF) is now generally used to measure the prestige of journals, the influence of articles published in those journals and, by extension, the authors, institutions and countries. ${ }^{2}$ In Brazil, the national agency (CNPQ) dedicated to promoting scientific and technological research has used the IF as an indicator of the national research output and to define investment priorities. ${ }^{3}$

In this paper, our goal was to identify the most frequently cited articles published by Brazilian authors in the most-cited international journals dedicated to general surgery. We aimed to provide an opportunity to recognize key historical advances and the evolution of surgery research in Brazil. 


\section{MATERIALS AND METHODS}

To identify the most frequently cited Brazilian papers published in general surgery journals, we accessed the Thompson Web of Knowledge database (Institute for Scientific Information - ISI). ${ }^{4}$ Inclusion criteria included articles with at least one Brazilian author and published in the 26 highly cited journals in the field of general surgery. These journals were selected based on their 2008 impact factor (Journal Citation Report). The search was limited to the period between January 1970 and June 2009.

The articles were identified and analyzed with respect to their authors, institution, year of publication, number of citations and topics.

An additional analysis was performed for articles that received more than 30 citations.

\section{RESULTS}

Of the 208,168 articles included in the study, 1,713 $(0.8 \%)$ were published by Brazilian authors. Nine were cited over 100 times, and these were considered classics. ${ }^{5}$ The 26 screened journals are listed in Table 1. Analyzing the ranking of each country's contributions, Brazilian authors achieved the $19^{\text {th }}$ overall position.

Brazilian contributions to those journals are listed by decades and presented in Table 2. Regarding Brazil's overall contribution, a rising curve was evident, with Brazil climbing from the $34^{\text {th }}$ position $(0.07 \%)$ in the seventies to the $13^{\text {th }}$ position in the 2000s (1.47\%). Figure 1 shows the number of Brazilian papers published in each year since 1970.

Because only nine Brazilian articles reached the classic denomination (article with more than 100 citations), we

Table 1 - Brazilian scientific contribution on the screened general surgery journals, during the period of 1970-2009 years.

\begin{tabular}{|c|c|c|c|c|c|}
\hline & & Impact factor & $\begin{array}{l}\text { Articles published } \\
\text { (n) }\end{array}$ & $\begin{array}{l}\text { Brazilian articles } \\
\text { published (n) }\end{array}$ & $\begin{array}{r}\text { Brazil (countries } \\
\text { ranking position) }\end{array}$ \\
\hline 1 & Annals of Surgery & 7.446 & 10.121 & $14(0.13 \%)$ & $29^{\text {th }}$ \\
\hline 2 & American Journal of Transplantation & 6.423 & 9.323 & $93(0.99 \%)$ & $14^{\text {th }}$ \\
\hline 3 & British Journal of Surgery & 4.304 & 23.992 & $24(0.10 \%)$ & $40^{\text {th }}$ \\
\hline 4 & American Journal of Surgical Pathology & 4.181 & 5.884 & $17(0.28 \%)$ & $29^{\text {th }}$ \\
\hline 5 & Endoscopy & 3.917 & 6.363 & $62(0.97 \%)$ & $23^{\text {rd }}$ \\
\hline 6 & Annals of Surgical Oncology & 3.917 & 5.457 & $30(0.54 \%)$ & $19^{\text {th }}$ \\
\hline 7 & Liver transplantation & 3.751 & 5.255 & $262(4.90 \%)$ & $7^{\text {th }}$ \\
\hline 8 & Transplantation & 3.641 & 22.092 & $107(0.48 \%)$ & $21^{\mathrm{st}}$ \\
\hline 9 & Archives of Surgery & 3.485 & 12.444 & $17(0.13 \%)$ & $27^{\text {th }}$ \\
\hline 10 & Shock & 3.325 & 5.677 & $100(1.76 \%)$ & $10^{\text {th }}$ \\
\hline 11 & Journal of the American College of Surgeons & 3.101 & 6.008 & $20(0.33 \%)$ & $15^{\text {th }}$ \\
\hline 12 & Surgery & 3.004 & 12.696 & $21(0.16 \%)$ & $29^{\text {th }}$ \\
\hline 13 & Obesity Surgery & 2.852 & 4.559 & $411(9.01 \%)$ & $3^{\text {rd }}$ \\
\hline 14 & Lasers in Surgery and Medicine & 2.700 & 5.390 & $135(0.27 \%)$ & $7^{\text {th }}$ \\
\hline 15 & Diseases of the Colon and Rectum & 2.621 & 10.420 & $67(0.64 \%)$ & $18^{\text {th }}$ \\
\hline 16 & Wound repair and regeneration & 2.445 & 1.846 & $14(0.75 \%)$ & $17^{\text {th }}$ \\
\hline 17 & Journal of burn care and rehabilitation & 2.421 & 801 & $4(0.49 \%)$ & $20^{\text {th }}$ \\
\hline 18 & Journal of Surgical Oncology & 2.384 & 5.660 & $26(0.45 \%)$ & $21^{\mathrm{st}}$ \\
\hline 19 & American Journal of Surgery & 2.337 & 13.389 & $46(0.34 \%)$ & $22^{\text {nd }}$ \\
\hline 20 & Journal of Trauma - Injury, Infection and Critical Care & 2.334 & 14.620 & $49(0.33 \%)$ & $22^{\text {nd }}$ \\
\hline 21 & Transplant International & 2.300 & 3.879 & $43(1.10 \%)$ & $21^{\text {st }}$ \\
\hline 22 & Journal of Gastrointestinal Surgery & 2.272 & 2.256 & $28(1.24 \%)$ & $12^{\text {th }}$ \\
\hline 23 & Surgical Endoscopy and other Interventional Techniques & 2.242 & 3.769 & $25(0.66 \%)$ & $24^{\text {th }}$ \\
\hline 24 & Clinical Transplantation & 1.923 & 2.320 & $37(1.59 \%)$ & $12^{\text {th }}$ \\
\hline 25 & Journal of Surgical Research & 1.836 & 7.881 & $21(0.26 \%)$ & $23^{\text {rd }}$ \\
\hline 26 & World Journal of Surgery & 1.778 & 6.066 & $40(0.66 \%)$ & $30^{\text {th }}$ \\
\hline Total & & & 208.163 & $1713(0.82 \%)$ & $19^{\text {th }}$ \\
\hline
\end{tabular}


Table 2 - The Brazilian most-cited articles in general surgery listed by periods, 1970-2009.

\begin{tabular}{lccc}
\hline Decade Period & $\begin{array}{c}\text { No Brazilian/total papers } \\
\text { published }(\%)\end{array}$ & $\begin{array}{c}\text { Brazil overall contributing } \\
\text { countries ranking }\end{array}$ & Papers cited $\geq 100$ times (n) \\
\hline $1970 / 1979$ & $17 / 22.348(0.07)$ & $34^{\text {th }}$ & - \\
$1980 / 1989$ & $71 / 34.940(0.20)$ & $28^{\text {th }}$ & $26^{\text {th }}$ \\
$1990 / 1999$ & $182 / 52.977(0.34)$ & $13^{\text {th }}$ & 5 \\
$2000 / 2009$ & $1.441 / 97.455(1.47)$ & $19^{\text {th }}$ & 3 \\
\hline Total & $1.713 / 207.720(0.82)$ & 9 \\
\hline
\end{tabular}

\section{Published items in each year}

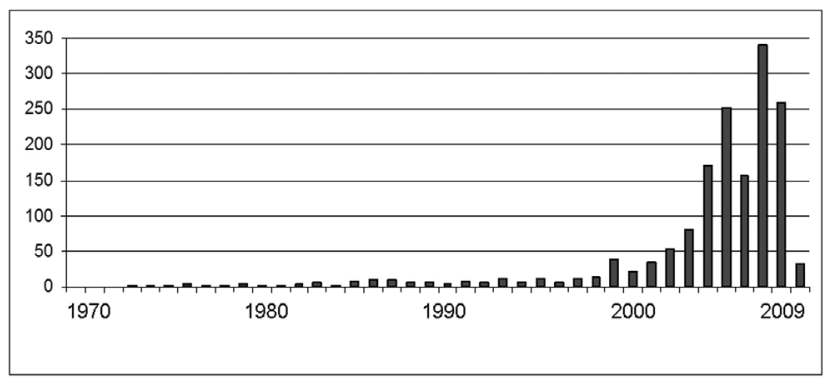

Figure 1 - Number of Brazilian articles published in The General Surgery Journals, during the period of 1970-2009.

further evaluated all of the articles with more than 30 citations (the top 65 articles). This cutoff was decided arbitrarily. Table 3 summarizes the designs of the 65 articles identified to have more than 30 citations. We observed a tendency for retrospective series and cohorts (56.9\%), while randomized trials accounted for 10/65 (15.3\%) articles. Twelve (18.4\%) experimental studies were included in the list of the top 65 .

Table 3 - The Brazilian most-cited articles by study design.

\begin{tabular}{lc}
\hline Study Design & $\begin{array}{c}\text { The Brazilian most } \\
\text { cited articles *(n) }\end{array}$ \\
\hline Meta-analysis & $1(1.5 \%)$ \\
Randomized clinical trials & $10(15.3 \%)$ \\
Experimental studies & $12(18.4 \%)$ \\
Case series/ cohorts & $37(56.9 \%)$ \\
Review articles & $5(7.7 \%)$ \\
Case reports & $0(0)$
\end{tabular}

$* \geq 30$ citations.

Topics covered by the "Brazilian Top 65" included oncology, transplantation, trauma and critical care, as listed in Table 4. Other topics were identified in this list, including gastrointestinal endoscopy and dentistry. The topics most frequently covered by the top 65 articles were shock and kidney transplantation at 10 articles each (15.3\%), followed by colorectal tumors in $9(13.8 \%)$ articles. Nineteen (29.2\%) articles covered themes related to oncology. The second most heavily cited theme was trauma/critical care and organ transplantation, accounting for 15/65 (22.9\%) articles each. Aside from these principal categories, bariatric surgery and GI endoscopy were covered in 4/65 (6.1\%) papers each (Table 4).

The 30 most-cited articles are presented in Table 5. The range of citations varied from 49 to 194, with an average of 85 citations per article. International collaboration was relevant since the earliest article to make the list, published in 1984 by

Table 4 - Topics covered.

\begin{tabular}{|c|c|c|}
\hline Topic & $\begin{array}{l}\text { Number of } \\
\text { articles (n) }\end{array}$ & $(\%)$ \\
\hline \multicolumn{3}{|l|}{ Oncology } \\
\hline Colorectal & 9 & 13.8 \\
\hline Gastric & 3 & 4.6 \\
\hline Renal pathology & 2 & 3.1 \\
\hline Neck tumors & 2 & 3.1 \\
\hline Eshopagus & 1 & 1.5 \\
\hline Sarcoma & 1 & 1.5 \\
\hline Breast & 1 & 1.5 \\
\hline \multicolumn{3}{|l|}{ Trauma and critical care } \\
\hline Shock & 10 & 15.3 \\
\hline Sepsis & 3 & 4.6 \\
\hline Abdominal compartment syndrome & 1 & 1.5 \\
\hline Penetrating abdominal wounds & 1 & 1.5 \\
\hline \multicolumn{3}{|l|}{ Organ Transplantation } \\
\hline Kidney & 10 & 15.3 \\
\hline Liver & 1 & 1.5 \\
\hline General & 4 & 6.1 \\
\hline Bariatric surgery & 4 & 6.1 \\
\hline \multicolumn{3}{|l|}{ Hepato-Biliary diseases } \\
\hline Laparoscopic cholecystectomy & 1 & 1.5 \\
\hline Portal hypertension & 1 & 1.5 \\
\hline Gastrointestinal endoscopy & 4 & 6.1 \\
\hline Dentistry & 2 & 3.1 \\
\hline Others & 3 & 4.6 \\
\hline Total (Top-65) & 65 & \\
\hline
\end{tabular}


Table 5 - List of the Brazilian thirty most cited articles in general surgery, during the period of 1970-2009 years.

\begin{tabular}{|c|c|c|c|c|c|c|c|c|}
\hline & $\begin{array}{c}\text { Number of } \\
\text { citations }\end{array}$ & Article & Year & Journal title & $1^{\text {st }}$ author & $\begin{array}{l}\text { Brazilian } \\
\text { Institutions }\end{array}$ & Country & $\begin{array}{l}\text { Brazilian authors } \\
\text { and co-authors }\end{array}$ \\
\hline 1 & 194 & $\begin{array}{l}\text { Restaging of colorectal cancer } \\
\text { based on the identification of } \\
\text { lymph node micrometastases } \\
\text { through immunoperoxidase stain- } \\
\text { ing of CEA and cytokeratins. }\end{array}$ & 1991 & $\begin{array}{l}\text { Dis Colon } \\
\text { Rectum }\end{array}$ & Cutait R, et al. & $\begin{array}{l}\text { USP } \\
\text { Hosp Sirio } \\
\text { Libanes }\end{array}$ & Brazil & $\begin{array}{l}\text { Cutait R, Alves } \\
\text { VA, Lopes LC, } \\
\text { Cutait DE, Borges } \\
\text { JL, Singer J, da } \\
\text { Silva JH, Goffi } \\
\text { FS. }\end{array}$ \\
\hline 2 & 140 & $\begin{array}{l}\text { CDX2, a highly sensitive and } \\
\text { specific marker of adenocarci- } \\
\text { nomas of intestinal origin - An } \\
\text { immunohistochemical survey } \\
\text { of } 476 \text { primary and metastatic } \\
\text { carcinomas. }\end{array}$ & 2003 & $\begin{array}{l}\text { Am J Surg } \\
\text { Patho }\end{array}$ & $\begin{array}{l}\text { Werling RW, } \\
\text { et al }\end{array}$ & $\begin{array}{l}\text { Botucatu } \\
\text { Unesp }\end{array}$ & USA/Brazil & Bacchi CE \\
\hline 3 & 130 & $\begin{array}{l}\text { The importance of thiopurine } \\
\text { methyltransferase activity for the } \\
\text { use of azathioprine in transplant } \\
\text { recipients. }\end{array}$ & 1992 & Transplantation & Chocair PR, et al & USP & Brazil/UK & Chocair PR \\
\hline 4 & 126 & $\begin{array}{l}\text { The influence of intraoperative } \\
\text { hypotension and perioperative } \\
\text { blood transfusion on disease-free } \\
\text { surviral in patients with complete } \\
\text { resection of colorectal liver me- } \\
\text { tastasis }\end{array}$ & 1991 & Ann Surg & Younes RN et al & USP & USA & Younes RN \\
\hline 5 & 125 & $\begin{array}{l}\text { Efficacy of hypertonic } 7.5 \% \text { saline } \\
\text { and } 6 \% \text { dextran- } 70 \text { in treating } \\
\text { trauma: a meta-analysis of con- } \\
\text { trolled clinical studies. }\end{array}$ & 1997 & Surgery & Wade CE, et al. & USP & USA/Brazil & Younes RN \\
\hline 6 & 112 & $\begin{array}{l}\text { Pit pattern in colorectal neoplasia: } \\
\text { Endoscopic magnifying view. }\end{array}$ & 2001 & Endoscopy & Kudo S, et al. & $\begin{array}{l}\text { Fugast Porto } \\
\text { Alegre }\end{array}$ & $\begin{array}{l}\text { Japan/ } \\
\text { Brazil/ } \\
\text { Sweden }\end{array}$ & Teixeira CR \\
\hline 7 & 103 & $\begin{array}{l}\text { Complications and hazards of } \\
\text { gastrointestinal endoscopy. }\end{array}$ & 1989 & World J Surg & $\begin{array}{l}\text { Habr-Gama A, } \\
\text { Waye JD }\end{array}$ & USP & Brazil/USA & Habr-Gama A \\
\hline 8 & 102 & $\begin{array}{l}\text { Prospective randomized trial of } \\
\text { mechanical bowel preparation } \\
\text { in patients undergoing elective } \\
\text { colorectal surgery. }\end{array}$ & 1994 & World J Surg & $\begin{array}{l}\text { Santos JCM, } \\
\text { et al. }\end{array}$ & $\begin{array}{l}\text { USP Ribeirao } \\
\text { Preto }\end{array}$ & Brazil & $\begin{array}{l}\text { Santos JCM, } \\
\text { Batista J, } \\
\text { Sirimarco MT, } \\
\text { Guimarães AS, } \\
\text { Levy CE. } \\
\end{array}$ \\
\hline 9 & 102 & $\begin{array}{l}\text { FTY720, a novel immunomodu- } \\
\text { lator: efficacy and safety results } \\
\text { from the first phase } 2 \text {. A study in } \\
\text { de novo renal transplantation. }\end{array}$ & 2005 & Transplantation & $\begin{array}{l}\text { Tedesco-Silva H, } \\
\text { et al. }\end{array}$ & UNIFESP & $\begin{array}{l}\text { Brazil/ } \\
\text { France/ } \\
\text { USA/... }\end{array}$ & Tedesco-Silva H \\
\hline 10 & 86 & $\begin{array}{l}\text { Low rectal cancer - Impact of } \\
\text { radiation and chemotherapy on } \\
\text { surgical treatment. }\end{array}$ & 1997 & $\begin{array}{l}\text { Dis Colon } \\
\text { Rectum }\end{array}$ & $\begin{array}{l}\text { Habr-Gama A, } \\
\text { et al. }\end{array}$ & USP & Brazil & $\begin{array}{l}\text { Habr-Gama A, } \\
\text { de Souza PMSB, } \\
\text { Ribeiro U, } \\
\text { Nadalin W, Gansl } \\
\text { R, Sousa AHSE, } \\
\text { Campos FG, } \\
\text { Gama-Rodrigues } \\
\text { J }\end{array}$ \\
\hline 11 & 86 & $\begin{array}{l}\text { Supraomohyoid neck dissection in } \\
\text { the treatment of } \mathrm{T} 1 / \mathrm{T} 2 \text { squamous } \\
\text { cell carcinoma of oral cavity. }\end{array}$ & 1994 & Am J Surgery & $\begin{array}{l}\text { Kligerman J, } \\
\text { et al. }\end{array}$ & INCA & Brazil & $\begin{array}{l}\text { Kligerman J, } \\
\text { Lima RA, Soares } \\
\text { JR, Prado L, Dias } \\
\text { FL, Freitas EQ, } \\
\text { Olivatto LO. }\end{array}$ \\
\hline 12 & 81 & $\begin{array}{l}\text { Operative versus nonoperative } \\
\text { treatment for stage } 0 \text { distal rectal } \\
\text { cancer following chemoradiation } \\
\text { therapy - Long-term results. }\end{array}$ & 2004 & Annals of Surg & $\begin{array}{l}\text { Habr-Gama A, } \\
\text { et al. }\end{array}$ & USP & Brazil & $\begin{array}{l}\text { Habr-Gama } \\
\text { A, Perez RO, } \\
\text { Nadalin W, } \\
\text { Sabbaga J, } \\
\text { Ribeiro U, Sousa } \\
\text { AHSE, Campos } \\
\text { FG, Kiss DR, } \\
\text { Gama-Rodrigues } \\
\text { J }\end{array}$ \\
\hline
\end{tabular}


Table 5 - List of the Brazilian thirty most cited articles in general surgery, during the period of 1970-2009 years. (cont.)

\begin{tabular}{|c|c|c|c|c|c|c|c|c|}
\hline & $\begin{array}{c}\text { Number of } \\
\text { citations }\end{array}$ & Article & Year & Journal title & $1^{\text {st }}$ author & $\begin{array}{l}\text { Brazilian } \\
\text { Institutions }\end{array}$ & Country & $\begin{array}{l}\text { Brazilian authors } \\
\text { and co-authors }\end{array}$ \\
\hline 13 & 81 & $\begin{array}{l}\text { Everolimus with optimized cyclo- } \\
\text { sporine dosing in renal transplant } \\
\text { recipients: 6-month safety and } \\
\text { efficacy results of two random- } \\
\text { ized studies. }\end{array}$ & 2004 & Am J Transp & Vitko S, et al. & $\begin{array}{l}\text { UNIFESP, } \\
\text { UNICAMP, } \\
\text { Santa Casa } \\
\text { Porto Alegre, } \\
\text { Hosp Geral } \\
\text { Fortaleza } \\
\end{array}$ & $\begin{array}{l}\text { Czech/ } \\
\text { Brazil/ } \\
\text { Spain/... }\end{array}$ & $\begin{array}{l}\text { Tedesco H, Alves } \\
\text { G, Garcia VD, } \\
\text { Esmeraldo R }\end{array}$ \\
\hline 14 & 80 & $\begin{array}{l}\text { A comparison of nonoperative vs. } \\
\text { preoperative radiotherapy in rectal } \\
\text { carcinoma. A } 10 \text {-year random- } \\
\text { ized trial. }\end{array}$ & 1989 & $\begin{array}{l}\text { Dis Colon } \\
\text { Rectum }\end{array}$ & $\begin{array}{l}\text { Reis Neto JA, } \\
\text { et al. }\end{array}$ & $\begin{array}{l}\text { PUC } \\
\text { Campinas }\end{array}$ & Brazil & $\begin{array}{l}\text { Reis Neto JA, } \\
\text { Quilici FA, Reis } \\
\text { JÁ }\end{array}$ \\
\hline 15 & 77 & $\begin{array}{l}\text { Primary renal synovial sarcoma: } \\
\text { molecular and morphologic de- } \\
\text { lineation of an entity previously } \\
\text { included among embryonal sar- } \\
\text { comas of the kidney. }\end{array}$ & 2000 & $\begin{array}{l}\text { Am J Surg } \\
\text { Patho }\end{array}$ & Argani $\mathrm{P}$, et al. & INCA & USA/Brazil & Faria PA \\
\hline 16 & 74 & $\begin{array}{l}\text { The role of lung innervation in the } \\
\text { hemodynamic response to hyper- } \\
\text { tonic sodium chloride solutions in } \\
\text { hemorrhagic shock. }\end{array}$ & 1985 & Surgery & Younes RN, et al. & USP & Brazil & Younes RN \\
\hline 17 & 72 & $\begin{array}{l}\text { Exposure-response relationships } \\
\text { for everolimus in de novo kidney } \\
\text { transplantation: defining a thera- } \\
\text { peutic range. }\end{array}$ & 2002 & Transplantation & $\begin{array}{l}\text { Kovarik JM, } \\
\text { et al. }\end{array}$ & UNIFESP & $\begin{array}{l}\text { Switzerland/ } \\
\text { USA/ } \\
\text { Brazil/... }\end{array}$ & Silva HAT \\
\hline 18 & 72 & $\begin{array}{l}\text { Ghrelin: a gut-brain hormone: } \\
\text { Effect of gastric bypass surgery }\end{array}$ & 2003 & Obes Surg & $\begin{array}{l}\text { Geloneze B, } \\
\text { et al. }\end{array}$ & UNICAMP & Brazil & $\begin{array}{l}\text { Geloneze B, } \\
\text { Tambascia } \\
\text { MA, Pilla VF, } \\
\text { Geloneze SR, } \\
\text { Repetto EM, } \\
\text { Pareja JC } \\
\end{array}$ \\
\hline 19 & 69 & $\begin{array}{l}\text { Diagnostic and therapeutic lapa- } \\
\text { roscopy for penetrating abdominal } \\
\text { trauma: A multicenter experience }\end{array}$ & 1996 & J Trauma & Zantut LF, et al. & USP & Brazil/USA & Zantut LF \\
\hline 20 & 68 & $\begin{array}{l}\text { Hypertonic solutions in the treat- } \\
\text { ment of hypovolemic shock: a } \\
\text { prospective, randomized study in } \\
\text { patients admitted to the emergency } \\
\text { room. }\end{array}$ & 1992 & Surgery & Younes RN, et al. & USP & Brazil & $\begin{array}{l}\text { Younes RN, Aun } \\
\text { F, Accioly CQ, } \\
\text { Casale LPL, } \\
\text { Szajnbok I, } \\
\text { Biroloni D. } \\
\end{array}$ \\
\hline 21 & 68 & $\begin{array}{l}\text { Simultaneous liver and lung injury } \\
\text { following gut ischemia is medi- } \\
\text { ated by xanthine oxidase. }\end{array}$ & 1991 & J Trauma & $\begin{array}{l}\text { Poggetti RS, } \\
\text { et al. }\end{array}$ & USP & USA/Brazil & Poggetti RS \\
\hline 22 & 64 & $\begin{array}{l}\text { Early gastric cancer: results in a } \\
\text { general hospital in Japan. }\end{array}$ & 1984 & World J Surg & Abe $S$, et al. & USP & Japan & $\begin{array}{l}\text { GamaRodrigues } \\
\text { JJ }\end{array}$ \\
\hline 23 & 64 & $\begin{array}{l}\text { Focal versus diffuse anaplasia in } \\
\text { Wilms tumor--new definitions } \\
\text { with prognostic significance: a } \\
\text { report from the National Wilms } \\
\text { Tumor Study Group. }\end{array}$ & 1996 & $\begin{array}{l}\text { Am J Surg } \\
\text { Patho }\end{array}$ & Faria P, et al. & INCA & USA/Brazil & Faria PA \\
\hline 24 & 63 & $\begin{array}{l}\text { Comparison of the low level laser } \\
\text { therapy effects on cultured human } \\
\text { gingival fibroblasts proliferation } \\
\text { using different irradiance and } \\
\text { same fluence. }\end{array}$ & 2001 & $\begin{array}{l}\text { Lasers in Surg } \\
\text { Med }\end{array}$ & $\begin{array}{l}\text { Almeida-Lopes } \\
\text { L, et al. }\end{array}$ & $\begin{array}{l}\text { USP } \\
\text { Univ Vale do } \\
\text { Paraiba }\end{array}$ & Brazil & $\begin{array}{l}\text { Almeida-Lopes L, } \\
\text { Rigau J, Zangaro } \\
\text { RA, Guidugli } \\
\text { Neto J, Jaeger } \\
\text { MM }\end{array}$ \\
\hline 25 & 60 & $\begin{array}{l}\text { Effect of low-power laser ir- } \\
\text { radiation on cell growth and } \\
\text { procollagen synthesis of cultured } \\
\text { fibroblasts }\end{array}$ & 2002 & $\begin{array}{l}\text { Lasers in Surg } \\
\text { Med }\end{array}$ & Pereira AN, et al. & USP & Brazil & $\begin{array}{l}\text { Pereira AN, } \\
\text { Eduardo CD, } \\
\text { Matson E, } \\
\text { Marques MM } \\
\end{array}$ \\
\hline 26 & 57 & $\begin{array}{l}\text { Ertapenem versus piperacillin/ } \\
\text { tazobactam in the treatment of } \\
\text { complicated intraabdominal infec- } \\
\text { tions: results of a double-blind, } \\
\text { randomized comparative phase } \\
\text { III trial. }\end{array}$ & 2003 & Annals of Surg & $\begin{array}{l}\text { Solomkin JS, } \\
\text { et al. }\end{array}$ & $\begin{array}{l}\text { Hosp } \\
\text { Evangelico } \\
\text { Curitiba }\end{array}$ & USA & Malafaia $\mathrm{O}$ \\
\hline
\end{tabular}


Table 5 - List of the Brazilian thirty most cited articles in general surgery, during the period of 1970-2009 years. (cont.)

\begin{tabular}{|c|c|c|c|c|c|c|c|c|}
\hline 27 & 57 & $\begin{array}{l}\text { Mycophenolate mofetil substitu- } \\
\text { tion for cyclosporine a in renal } \\
\text { transplant recipients with chronic } \\
\text { progressive allograft dysfunction: } \\
\text { the "creeping creatinine" study. }\end{array}$ & 2005 & Transplantation & Dudley C, et al. & UNIFESP & $\begin{array}{l}\text { UK/Austria/ } \\
\text { Czech/ } \\
\text { Switzerland/ } \\
\text { Brazil }\end{array}$ & Silva HAT \\
\hline 28 & 54 & $\begin{array}{l}\text { Individual patient cohort analysis } \\
\text { of the efficacy of hypertonic } \\
\text { saline/dextran in patients with } \\
\text { traumatic brain injury and hypo- } \\
\text { tension. }\end{array}$ & 1996 & J Trauma & Wade CE, et al. & USP & $\begin{array}{l}\text { Sweden/ } \\
\text { USA/Brazil }\end{array}$ & Younes RN \\
\hline 29 & 51 & $\begin{array}{l}\text { Everolimus versus mycophenolate } \\
\text { mofetil in the prevention of rejec- } \\
\text { tion in de novo renal transplant } \\
\text { recipients: a } 3 \text {-year randomized, } \\
\text { multicenter, phase III study. }\end{array}$ & 2005 & Transplantation & Lorber MI, et al. & UNIFESP & $\begin{array}{l}\text { USA/ } \\
\text { Switzerland/ } \\
\text { Brazil }\end{array}$ & Tedesco H \\
\hline 30 & 51 & $\begin{array}{l}\text { Results of a prospective trial on } \\
\text { elective modified radical classi- } \\
\text { cal versus supraomohyoid neck } \\
\text { dissection in the management } \\
\text { of oral squamous carcinoma. } \\
\text { Brazilian Head and Neck Cancer } \\
\text { Study Group. }\end{array}$ & 1998 & Am J Surg & $\begin{array}{l}\text { Brentani RR, } \\
\text { et al. }\end{array}$ & $\begin{array}{l}\text { Hosp AC } \\
\text { Camargo, } \\
\text { Hosp Araujo } \\
\text { Jorge, } \\
\text { USP, } \\
\text { Hosp Erasto } \\
\text { Gaertner, } \\
\text { Hosp } \\
\text { Heliopolis, } \\
\text { Hosp } \\
\text { Napoleao } \\
\text { Laureano, } \\
\text { UNIFESP, } \\
\text { Santa Casa } \\
\text { Porto Alegre }\end{array}$ & Brazil & $\begin{array}{l}\text { Brazilian Head } \\
\text { and Neck Cancer } \\
\text { Study Group }\end{array}$ \\
\hline
\end{tabular}

Abe $\mathrm{S}$ et al. (Gama-Rodrigues), described early gastric cancer in Japan. ${ }^{6}$ The most recent classic (102 citations), published by Tedesco-Silva et al. in 2005, was a phase II international clinical trial describing the immunologic response to FTY720 in kidney transplantation. ${ }^{7}$ Indeed, eighteen articles (60\%) on this list were written with international co-authorship. The article ranked in first place on the list was published in the nineties by Cutait et al. and discussed colorectal cancer staging based on immunohistochemical analysis of micrometastasis in lymph nodes. ${ }^{8}$

Analyzing the individual contribution by state, institutions from Sao Paulo authored or co-authored $65 \%$ of these articles. The most-published institutions were the University of Sao Paulo/Hospital de Clinicas (USP), the Federal University of Sao Paulo (UNIFESP), Campinas State University (UNICAMP) and Albert Einstein Hospital, contributing 619, 173, 87 and 87 papers, respectively. Nevertheless, notable contributions came from others states, such as Rio de Janeiro, Rio Grande do Sul, Minas Gerais and Parana (Table 6).

\section{DISCUSSION}

Eventually, all surgical specialties are dependent on general surgery. General surgery is a discipline encompassing clinical knowledge, operative techniques and scientific foundations, including wound healing, infection, metabolism, immunology, shock and resuscitation.

In this paper, we recognized articles that were published in the most cited general surgery journals, had at least one author with a Brazilian affiliation, were identified in the ISI Web of Knowledge database and were published during a 39-year period (1970 to 2009). In total, 1,713 items were published by Brazilian authors from more than 100 institutions. It is worth mentioning that 555 papers received 5,556 citations during this period. Therefore, 1,158 (67.6\%) articles remained uncited. In fact, approximately one half of all published medical articles are never subsequently cited.

A limitation of our results is that we have no information on citations in journals not covered by the ISI bibliometric database. Undoubtedly, the results of this citation analysis could be different if more Latin American scientific journals were listed in the ISI Web of Knowledge or if another citation database, such as SCOPUS, had been selected. Another aspect that must be taken into consideration is that, until recently, only articles published in English were considered by the JCR.

The 26 screened journals were the periodicals dedicated to general surgery with the highest impact factor. These international journals receive contributions from all over the 
Table 6 - Brazilian most-cited articles in general surgery, sorted by the top contributing Institutions.

\begin{tabular}{|c|c|c|c|}
\hline Institutions & $\begin{array}{c}\text { Number of articles } \\
\text { published }(\%)\end{array}$ & $\begin{array}{l}\text { Number of articles with } \\
\text { more than } 30 \text { citations (\%) }\end{array}$ & $\begin{array}{l}\text { Number of classical } \\
\text { articles published (\%) }\end{array}$ \\
\hline USP - Hosp Clin SP & $619(36.1)$ & $36(56.2)$ & $5(55.5)$ \\
\hline UNIFESP - Escola Paulista & $173(10.1)$ & $8(12.5)$ & $1(11.1)$ \\
\hline Hospital Sirio Libanes & $14(0.8)$ & $2(3.1)$ & $1(11.1)$ \\
\hline USP Ribeirao Preto & $10(1.4)$ & $1(1.5)$ & $1(11.1)$ \\
\hline INCA & $13(0.7)$ & $4(6.2)$ & 0 \\
\hline UFMG & $53(3.1)$ & $3(4.7)$ & 0 \\
\hline UFRJ & $51(2.9)$ & $3(4.7)$ & 0 \\
\hline Santa Casa Porto Alegre - UFCSPA & $30(1.7)$ & $3(4.7)$ & 0 \\
\hline UNICAMP & $87(5.1)$ & $2(3.1)$ & 0 \\
\hline UFRGS & $63(3.6)$ & $2(3.1)$ & 0 \\
\hline Hospital AC Camargo & $55(3.2)$ & $1(1.5)$ & 0 \\
\hline PUCRS & $22(1.3)$ & $1(1.5)$ & 0 \\
\hline Fundação Oswaldo Cruz & $8(0.4)$ & $1(1.5)$ & 0 \\
\hline Hospital Albert Einstein & $87(5.1)$ & 0 & 0 \\
\hline Gastro Obeso Center & $36(2.1)$ & 0 & 0 \\
\hline Bonsucesso General Hospital & $30(1.7)$ & 0 & 0 \\
\hline Univ Est Londrina & $25(1.4)$ & 0 & 0 \\
\hline UFPR & $13(0.7)$ & 0 & 0 \\
\hline UERJ & $12(0.7)$ & 0 & 0 \\
\hline Hospital Beneficiencia Portuguesa & $9(0.5)$ & 0 & 0 \\
\hline Others/Unknow & $322(18.7)$ & $13(20.3)$ & $2(22.2)^{*}$ \\
\hline
\end{tabular}

* - Botucatu Unesp and Fugast contributed with 1 classical article each. USP/Hosp Clin SP - Universidade de São Paulo/ Hospital de Clinicas; UNIFESP Universidade Federal de São Paulo; INCA - Instituto Nacional do Câncer; UFMG - Universidade Federal de Minas Gerais; UFRJ - Universidade Federal do Rio de Janeiro; UFCSPA - Universidade Federal de Ciências da Saúde de Porto Alegre; UNICAMP - Universidade Estadual de Campinas; UFRGS - Universidade Federal do rio Grande do Sul; Hosp AC Camargo - Hospital AC Camargo and Ludwig Institute of Cancer Research; PUC/RS - Pontificia Universidade Católica do Rio Grande do Sul; UEL - Universidade Estadual de Londrina; UFPR - Universidade Federal do Paraná; UERJ - Universidade Estadual do Rio de Janeiro.

world, publishing milestone publications in experimental and clinical surgery.

Although English is not the native language in Brazil, our country has an established place in the general surgery literature. Nowadays, Brazilian authors have the $19^{\text {th }}$ overall position in terms of the number of papers published in these renowned journals. The contribution of papers from Brazilian authors in these journals is encouraging, as the proportion of the global total increased by about 21 -fold, from $0.07 \%$ in the 1970 s to $1.47 \%$ in the 2000 s. Nonetheless, Brazil is behind small developing countries, such as Taiwan, and it just recently surmounted Turkey. The overwhelming supremacy of United States was evident, as the United States was the top contributing country in 23 of 26 screened journals, publishing more than $52 \%$ of all papers.

In the 2000s, Brazil occupied the $13^{\text {th }}$ overall position. In our point of view, this ascension can be partially attributed to the support of the Brazilian National Council, which is dedicated to promoting science in our country. In addition, more international exchange opportunities, widespread use of the internet and more surgeons dedicated to basic and clinical research could be responsible for the rising curve of publications over the years. ${ }^{9}$ However, the English language can be a barrier, and other types of biases (bias towards nationality) can influence the approval rate. ${ }^{2} \mathrm{We}$ recognize that the SciELO (Scientific Electronic Library Online) database increased the visibility of Brazilian medical literature during the past decade. The SciELO will probably also contribute to increasing the impact of national journals devoted to surgery.

Specific topics were highlighted in different decades. These data reflect, at least in part, the evolution of a specific surgical area. For example, a better comprehension of the physiology of obesity, transplantation and laser therapy resulted in great amounts of new information and motivated the establishment of new journals. Our study shows that many Brazilian papers are devoted to these 
topics. Concerning bariatric surgery, Brazilian authors have published continually in journals like Obesity Surgery, where Brazil holds the $3^{\text {rd }}$ overall position.

Various observations can be made regarding the study design of the top articles. We observed a tendency of clinically oriented publications. Only $12(18.4 \%)$ of the top 65 articles presented an experimental design. Although surgeons are conscious of the importance of evidence-based medicine, only 10 (15.3\%) articles were randomized clinical trials. Prior studies corroborated these observations in other medical areas, including trauma, general surgery, urology and critical care medicine. , $^{50-12}$

Interestingly, international collaboration and multicenter trials seem to be needed for a paper to be highly cited because 7 of the 9 classics (> 100 citations) have authors from different countries. In the 1980s, 9/71 (12.6\%) articles had inter-institutional collaboration. In the 1990s, 32/182 (17.1\%) articles published by Brazilian authors had this characteristic, and after 2000, 185 of 1441 (12.8\%) articles had international collaboration. Eighteen of the top 30 Brazilian articles were inter-institutional. In the top 65 articles ( $>30$ citations), the United States was the country whose investigators most frequently collaborated with Brazilian authors $(18 / 65 ; 27.7 \%)$.

General surgery is a heterogeneous medical specialty, covering different areas in men and women's health, basic science, clinical practice and operative techniques. The list of the top-cited articles covers some of the hottest topics in surgery today, such as surgical oncology, minimally invasive approaches and organ transplantation. In the most cited article concerning trauma and critical care, Wade et al. from the University of California and in collaboration with Younes from USP published a meta-analysis demonstrating similar efficacy between hypertonic and isotonic solutions in trauma. Previously, Younes et al. reported a cohort of Memorial SloanKettering patients and analyzed perioperative risk factors and the recurrence of colorectal metastases ( $4^{\text {th }}$ overall). The same author published the best-ranked Brazilian experimental study concerning the use of hypertonic saline in dogs (positioned $\left.16^{\text {th }}\right)$. The top article covering organ transplantation was presented by Chocair et al. (USP) and covered the activity of thiopurine methyltransferase in immunosuppressive therapy in renal transplantation. The article ranked first overall and in oncology was published by Cutait et al. (USP - Hospital Sirio Libanes) and was about immunohistology in colorectal cancer.
The oldest article in the list of the top 65 was published in 1979 by Paulino et al. (UFRJ) regarding early gastric cancer. The most recent top 65 article was published by Zorron et al. (RJ) in 2008, describing preliminary results of transvaginal NOTES cholecystectomy.

In the $7^{\text {th }}$ most cited article, Habr-Gama et al. (USP) reviewed the complications of GI endoscopy. The same group addressed the use of radiotherapy and chemotherapy in low rectal cancer $\left(10^{\text {th }}\right)$ and more recently published two articles reporting the long-term results of operative treatment for rectal cancer following chemotherapy $\left(14^{\text {th }}\right.$ and $\left.60^{\text {th }}\right)$.

Although our search included the 26 most-cited general surgery journals, we are aware that restricting our search to these journals is a limitation of the present study. Some of the most cited Brazilian papers were published in more specific or general journals; these include the description of the "Jatene procedure" for the correction of transposition of the great arteries, which was published in the Journal of Thoracic and Cardiovascular Surgery in 1976; the use of hypertonic $\mathrm{NaCl}$ for the treatment of severe hemorrhagic shock, which was published in the American Journal of Physiology in 1980; and the first description of liver transplantation from a living donor, which was published by Raia et al. in 1989 in the Lancet. These articles have been cited 243, 258 and 262 times, respectively. ${ }^{13-15}$

This analysis highlighted the academic influence and popularity of southeast institutions. Historically, Sao Paulo institutions provided financial and technological support for research. For decades, the University of Sao Paulo - Hospital de Clinicas has been the top published institution. However, highly contributing institutions are now also based in other states, such as Rio de Janeiro, Rio Grande do Sul, Minas Gerais and Parana.

\section{CONCLUSIONS}

In recent years, citation analysis, although imperfect, has become common and is considered to be the currency of journal prestige. The selected journals have always attracted the submission of advances and landmark articles in general surgery. This study is an opportunity to remember the mostcited Brazilian articles and to learn from distinguished Brazilian surgeons, recognizing the remarkable impact that these visionaries have had in national surgery. 


\section{REFERENCES}

1. Garfield E. Citation indexing for studying science. Nature. 1970;227:66971.

2. Callaham M, Wears RL, Weber E. Journal prestige, publication bias, and other characteristics associated with citation of published studies in peer-reviewed journals. Jama. 2002;287:2847-50.

3. Rocha-e-Silva M. The new Qualis, or the announced tragedy. Clinics. 2009;64:1-4.

4. ISI Web of knowledge database. Available at: http://apps.isiknowledge. com.

5. Heldwein FL, Rhoden EL, Morgentaler A. Classics of Urology: A Half Century History of the Most Frequently Cited Articles (1955-2009). Urology in press.

6. Abe S, Ogawa Y, Nagasue N, Sasaki Y, Akamizu H, Hirose S, et al. Early gastric cancer: results in a general hospital in Japan. World J Surg. 1984;8:308-14

7. Tedesco-Silva H, Mourad G, Kahan BD, Boira JG, Weimar W, Mulgaonkar S, et al. FTY720, a novel immunomodulator: efficacy and safety results from the first phase $2 \mathrm{~A}$ study in de novo renal transplantation. Transplantation. 2005;79:1553-60.

8. Cutait R, Alves VA, Lopes LC, Cutait DE, Borges JL, Singer J, et al. Restaging of colorectal cancer based on the identification of lymph node micrometastases through immunoperoxidase staining of CEA and cytokeratins. Dis Colon Rectum. 1991;34:917-20.
9. Goldenberg S. Part of the history of surgical research in Brazil: historical evolution of the journal Acta Cirurgica Brasileira (1986-2005). Acta Cir Bras. 2008;23:1-3.

10. Ollerton JE, Sugrue M. Citation classics in trauma. J Trauma. 2005;58:364-9.

11. Garfield E. 100 citation classics from the Journal of the American Medical Association. Jama. 1987;257:52-9.

12. Paladugu R, Schein M, Gardezi S, Wise L. One hundred citation classics in general surgical journals. World J Surg. 2002;26:1099-105.

13. Jatene AD, Fontes VF, Paulista PP, Souza LC, Neger F, Galantier M, et al. Anatomic correction of transposition of the great vessels. J Thorac Cardiovasc Surg. 1976;72:364-70.

14. Velasco IT, Pontieri V, Rocha e Silva M Jr, Lopes OU. Hyperosmotic $\mathrm{NaCl}$ and severe hemorrhagic shock. Am J Physiol. 1980;239:H664-73

15. Raia S, Nery JR, Mies S. Liver transplantation from live donors. Lancet. 1989;2:497. 